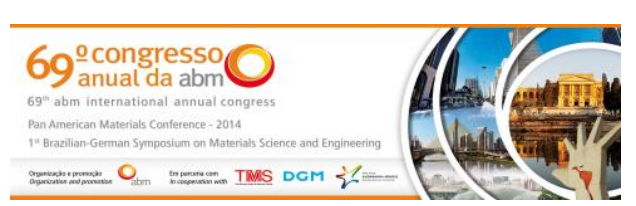

Tema: Materiais cerâmicos, compósitos e poliméricos

\title{
USO DE ARGILA ORGANOFÍLICA COMO ADITIVO NUCLEANTE PARA POLIPROPILENO*
}

\author{
Albaniza Alves Tavares ${ }^{1}$ \\ Diêgo Fabrício Alcântara Silva² \\ Daniela de Lourdes Anjos Coutinho Simões Andrade ${ }^{3}$ \\ Claudia Maria de Oliveira Raposo 4 \\ Eduardo Luís Canedo 5 \\ Suédina Maria de Lima Silva ${ }^{6}$
}

\section{Resumo}

Neste estudo, a influência do tipo de argila como nucleante heterogêneo para o PP foi avaliada. As argilas organofílicas MPH e MPTH, provenientes do Laboratório de físico-química da UAMG/UFCG, e C20A da Southern Clay Products, em teor de 1\% em massa, foram misturadas com o polipropileno (PP) compatibilizado com $15 \%$ em massa de polipropileno enxertado com anidrido maleico (PP-g-MA), em misturador interno do reômetro de torque Haake visando a obtenção de concentrados que posteriormente foram diluídos com o PP em extrusora. As amostras preparadas foram caracterizadas por medidas de calorimetria exploratória diferencial (DSC) e difratometria de raios- $\mathrm{X}$ (DRX). Os resultados obtidos mostraram que embora as três argilas tenham atuado como agente nucleante para o PP a MPH foi a mais eficiente. Palavras-chave: PP; Argila organofílica; Agente nucleante.

\section{USE OF ORGANOCLAY AS NUCLEATING ADDITIVE FOR POLYPROPYLENE}

\begin{abstract}
In this study, the influence of the type of clay as a heterogeneous nucleant for PP was evaluated. The organoclays MPH and MPTH, from the Laboratory of Physical and Chemical UAMG / UFCG and C20A from Southern Clay Products in content of 1 mass $\%$, were mixed with polypropylene (PP) compatibilized with $15 \mathrm{wt} \%$ of polypropylene grafted with maleic anhydride (PP-g-MA) in the torque rheometer Haake internal mixer in order to obtain concentrates were subsequently diluted with PP extruder. The prepared samples were characterized by differential (DSC) scanning calorimetry and X-ray diffractometry (XRD). The results showed that although the three clays have acted as nucleating agent for PP MPH was the most efficient.
\end{abstract}

Keywords: PP; Organoclay; Nucleating agent.

1 Pós-Graduanda, Programa de Pós Graduação em Ciências e Engenharia de Materiais, UFCG, Campina Grande, $P B$, Brasil.

2 Graduando, Departamento de Engenharia de Materiais, UFCG, Campina Grande, PB, Brasil

3 Eng a de Materiais, Doutora, Professora Departamento de Engenharia de Materiais, UFCG, Campina Grande, PB, Brasil.

4 Enga Química, Doutora, Professora Departamento de Mineração e Geologia, UFCG, Campina Grande, PB, Brasil

5 Engo Químico, Doutor, Pesquisador do Instituto de Tecnologia de Pernambuco (ITEP), Recife, PE, Brasil.

6 Eng a de Materiais, Doutora, Professora Departamento de Engenharia de Materiais, UFCG, Campina Grande, PB, Brasil.

\footnotetext{
* Contribuição técnica ao $69^{\circ}$ Congresso Anual da ABM - Internacional e ao 14ํㅡㄹ ENEMET - Encontro Nacional de Estudantes de Engenharia Metalúrgica, de Materiais e de Minas, 21 a 25 de julho de 2014, São Paulo, SP, Brasil.
} 


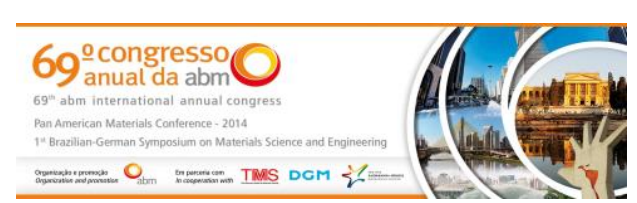

\section{INTRODUÇÃO}

A combinação atrativa de baixo custo, fácil processabilidade e bom balanço de propriedades faz do polipropileno um dos termoplásticos de maior consumo mundial. A grande versatilidade deste polímero, seja na forma pura ou reforçada, permite ampla gama de aplicação em fibras, filmes e artefatos moldados [1]. Entretanto, apresentam desvantagens tais como baixa propriedade de barreira ao oxigênio, baixa estabilidade térmica e dimensional que podem limitar seu uso em certas aplicações [2].

Uma estratégia para melhorar as propriedades do PP é através do desenvolvimento de nanocompósitos polímeros/silicato em camadas. Esta abordagem tem atraído grande interesse acadêmico e industrial devido à possibilidade de melhorar propriedades mecânicas, térmicas, elétricas e de barreira a baixos níveis de carregamento ( $1 \mathrm{a} 5 \%$ em massa de argila organofílica) sem comprometimento da processabilidade e da densidade do material, fatores essenciais para a indústria de embalagens [3]. Além disso, alguns estudos têm demonstrado que uma pequena quantidade de argila organofílica quando bem dispersa pode atuar como agente nucleante eficaz para acelerar a cristalização do polipropileno (PP) e neste caso promover o melhoramento das propriedades mecânicas devido à formação de cristais mais perfeitos e ao aumento no número de moléculas atadoras [4-8].

Nanocompósitos polímero/silicato em camadas podem ser preparados por três rotas: intercalação por solução, polimerização in situ e intercalação por fusão, sendo esta última a preferida por dispensar o uso de solventes (reduzindo o número de etapas, riscos ambientais e custo) bem como possibilitar o uso de equipamentos de processamento convencionais como extrusoras e injetoras [9]. Embora estudos sobre nanocompósitos PP/argila organofílica tenham sido realizados, as propriedades dos nanocompósitos obtidos são fortemente dependentes das características da argila.

Entre os fatores que governam o melhoramento das propriedades podemos citar o grau de esfoliação da argila em presença do polímero e a afinidade entre ambos [10]; a escolha correta da argila modificada [11]; e o seu grau de pureza, visto que a presença de contaminantes, como a matéria orgânica presente na bentonita, pode intervir na conversão da argila natural em organofílica e consequentemente reduzir as interações polímero/argila e assim a extensão de intercalação ou esfoliação no nanocompósito final. Além disso, as argilas organofílicas empregadas no desenvolvimento dos nanocompósitos PP/argila reportados na literatura são do tipo comerciais, frequentemente provenientes da National Lead Industries, Sud-Chemie e Southern Clay Products, e, portanto de custo muito elevado o que pode inviabilizar a aplicação comercial destes nanocompósitos poliméricos em indústrias brasileiras [12]. Neste estudo a proposta foi utilizar duas argilas bentonitas preparadas com tecnologia nacional no laboratório de Físico-química da Unidade Acadêmica de Mineração e Geologia da UFCG (MPH e MPTH) e uma comercial (C20A) na preparação de nanocompósitos de polipropileno (PP) e avaliar o uso destas como aditivo nucleante heterogêneo para o PP.

\footnotetext{
* Contribuição técnica ao $69^{\circ}$ Congresso Anual da ABM - Internacional e ao 14ํㅡㄹ ENEMET - Encontro Nacional de Estudantes de Engenharia Metalúrgica, de Materiais e de Minas, 21 a 25 de julho de 2014, São Paulo, SP, Brasil.
} 


\section{MATERIAIS E MÉTODOS}

\subsection{Materiais}

As argilas organofílicas empregadas neste estudo foram: a montmorilonita organofílica Cloisite 20A (C20A), fornecida pela Southern Clay Products, Texas/EUA, com capacidade de troca de cátions (CTC) de 0,95 meq/g de argila [13] e as bentonitas organofílicas (MPH e MPTH), fornecidas pelo Laboratório de Físicoquímica da Unidade Acadêmica de Mineração e Geologia da UFCG/Campina Grande/PB, com CTC de 0,92 meq/g de argila. O polipropileno (PP) HP525M (índice de fluidez de $8 \mathrm{~g} / 10 \mathrm{~min}$ a $230^{\circ} \mathrm{C} / 2,16 \mathrm{Kg}$ - ASTM D 1238), produzido pela Quattor e fornecido pela Felinto Indústria e Comércio LTDA, na forma de pellets, foi empregado como matriz polimérica na preparação dos filmes dos nanocompósitos [14]. Polipropileno modificado com anidrido maléico (PGA), de nome comercial Polybond ${ }^{\circledR} 3150$ (índice de fluidez de $50 \mathrm{~g} / 10 \mathrm{~min}$ a $230^{\circ} \mathrm{C} / 2,16 \mathrm{Kg}$, temperatura de fusão de $157^{\circ} \mathrm{C}$, nível de $0,5 \%$ em massa de anidrido maléico), fornecido pela Chemtura Indústria Química do Brasil [15] foi utilizado como compatibilizante na preparação dos nanocompósitos PP/argila organofílica.

\subsection{Métodos}

Inicialmente concentrados (masterbatchs) de PGA e argila organofílica (C20A, MPH e MPTH) foram preparados na proporção 3:1, em um misturador interno (Reomix 600 ) do reômetro de torque Haake-Büchler System 90, operando com rotores do tipo roller, a uma temperatura de $170^{\circ} \mathrm{C}$ e velocidade de $60 \mathrm{rpm}$ durante 12 minutos. Em seguida os concentrados foram triturados, secos $\left(80^{\circ} \mathrm{C} / 24 \mathrm{~h}\right)$ e posteriormente diluídos no PP em extrusora dupla rosca contra rotativa do reômetro de torque Haake, em quantidade que resultou em híbridos com 1\% em massa de argila e 15\% em massa de PGA. As amostras extrusadas foram trituradas, secas $\left(80^{\circ} \mathrm{C} / 24 \mathrm{~h}\right)$ e alimentadas em extrusora Chill-Roll 16 de filme plano da AX Plásticos, operando com zonas de aquecimento estabelecidas num gradiente de 196 a $206^{\circ} \mathrm{C}$, visando obter filmes planos. Os filmes de PP puro e da matriz polimérica foram codificados de PP e PP/PGA, respectivamente e os concentrados e os híbridos na forma extrusada e de filmes estão listados na Tabela 1.

Tabela 1. Codificação dos concentrados e dos híbridos

\begin{tabular}{cccccc}
\hline $\begin{array}{c}\text { Amostra } \\
\text { (Concentrado) }\end{array}$ & Código & $\begin{array}{c}\text { Amostra } \\
\text { (Extrusado) }\end{array}$ & Código & Amostra (Filme) & Código \\
\hline PGA/C20A & CC20A & PP/PGA/C20A & HEC20A & PP/PGA/C20A & HFC20A \\
PGA/MPH & CMPH & PP/PGA/MPH & HEMPH & PP/PGA/MPH & HFMPH \\
PGA/MPTH & CMPTH & PP/PGA/MPTH & HEMPTH & PP/PGA/MPTH & HFMPTH \\
\hline
\end{tabular}

\subsubsection{Caracterização}

As medidas de DRX foram realizadas em equipamento Shimadzu XDR 7000 a 40kV e $30 \mathrm{~mA}$, com comprimento de onda $\lambda \mathrm{CuK} \alpha=1,5418 \AA$, em um intervalo de $2 \theta$ entre 2,0 e $12,0^{\circ}$ e velocidade de varredura de $2^{\circ} / \mathrm{min}$. As análises de calorimetria exploratória diferencial (DSC) foram realizadas em um calorímetro, modelo Q20, da TA Instruments, utilizando cadinho de alumínio fechado sob atmosfera de nitrogênio

\footnotetext{
* Contribuição técnica ao $69^{\circ}$ Congresso Anual da ABM - Internacional e ao 14ํㅡㄹ ENEMET - Encontro Nacional de Estudantes de Engenharia Metalúrgica, de Materiais e de Minas, 21 a 25 de julho de 2014, São Paulo, SP, Brasil.
} 


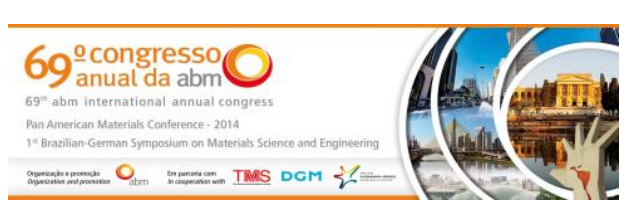

é que a carga inorgânica pode favorecer ao empilhamento ou ao ordenamento das cadeias de PP para o crescimento dos cristalitos [21].

De acordo com a Figura 2 e Tabela 2, os valores de Tc para os nanocompósitos praticamente não variaram em função do tipo de argila. Além disso, a incorporação destas argilas a matriz polimérica PP/PGA resultou em um aumento de apenas $3{ }^{\circ} \mathrm{C}$. Os dados de DRX apresentados na Figura 1 sugerem que a área superficial das partículas de argila disponível para interações no PP/PGA/argila deve ter sido a mesma para os três nanocompósitos resultando em um efeito nucleante similar.

É sabido que se a argila estiver dispersa dentro da matriz polimérica em escala nanométrica, onde os tamanhos das partículas de argila se encontram próximos aos das cadeias poliméricas, o molhamento das partículas de argila pelas cadeias poliméricas pode ser pouco favorecido e neste caso a argila atuará como fraco agente de nucleação heterogêneo para o polímero.

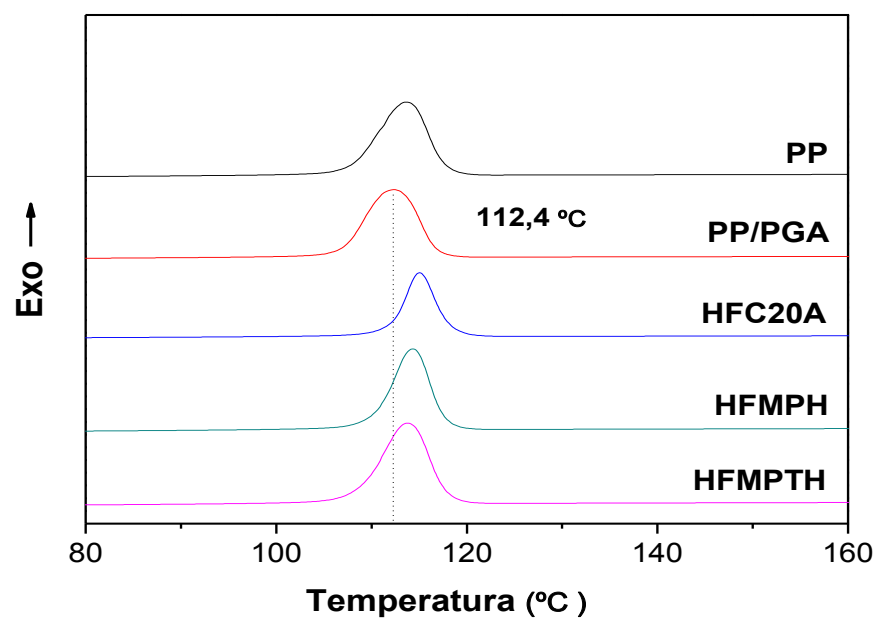

Figura 2. Curvas de DSC a uma taxa de resfriamento de $10^{\circ} \mathrm{C} / \mathrm{min}$.

Tabela 2. Dados obtidos das curvas de DSC

\begin{tabular}{|c|c|c|c|c|c|c|}
\hline Parâmetro & Unidade & PP & PP/PGA & HFC20A & HFMPH & HFMPTH \\
\hline $\begin{array}{l}\text { Temperatura do pico } \\
\text { de cristalização }\end{array}$ & $\left({ }^{\circ} \mathrm{C}\right)$ & 113,7 & 112,4 & 115,6 & 115,1 & 113,8 \\
\hline Cristalinidade & $(\%)$ & 55,3 & 43,6 & 40,0 & 48,8 & 42,5 \\
\hline $\begin{array}{l}\text { Taxa máxima de } \\
\text { cristalização }\end{array}$ & $\left(\min ^{-1}\right)$ & 1,513 & 1,614 & 1,842 & 1,751 & 1,514 \\
\hline $\begin{array}{l}\text { Tempo para cristalizar } \\
50 \% \text { do total }\end{array}$ & $(\min )$ & 0,92 & 1,02 & 0,88 & 0,87 & 0,91 \\
\hline
\end{tabular}

A partir dos dados de cristalização não isotérmica obtidos por DSC, a cristalinidade relativa, como uma função da temperatura, do PP puro, da matriz PP/PGA e dos nanocompósitos PP/PGA/argila organofílica, foi determinada usando a Equação 1.

$$
X_{t}=\int_{T_{0}}^{T}\left(d H_{c} / d T\right) / \int_{T_{0}}^{T_{\infty}}\left(d H_{c} / d T\right) d T
$$

\footnotetext{
* Contribuição técnica ao $69^{\circ}$ Congresso Anual da ABM - Internacional e ao 14ํㅡㄹ ENEMET - Encontro Nacional de Estudantes de Engenharia Metalúrgica, de Materiais e de Minas, 21 a 25 de julho de 2014, São Paulo, SP, Brasil.
} 
Onde $T_{0}$ e To são as temperaturas de cristalização inicial e final, respectivamente e $d H c / d T$ é o fluxo de calor.

A Figura 3 mostra que nos intervalos entre 10 e $90 \%$ de cristalinidade relativa, os nanocompósitos (HFC20A, HFMPH e HFMPTH) alcançaram estes percentuais em temperaturas mais altas do que a matriz polimérica pura (PP/PGA) submetida às mesmas condições de processamento. Isto confirmando a atuação das argilas (C20A, MPH e MPTH) como nucleantes heterogêneos para o PP/PGA.

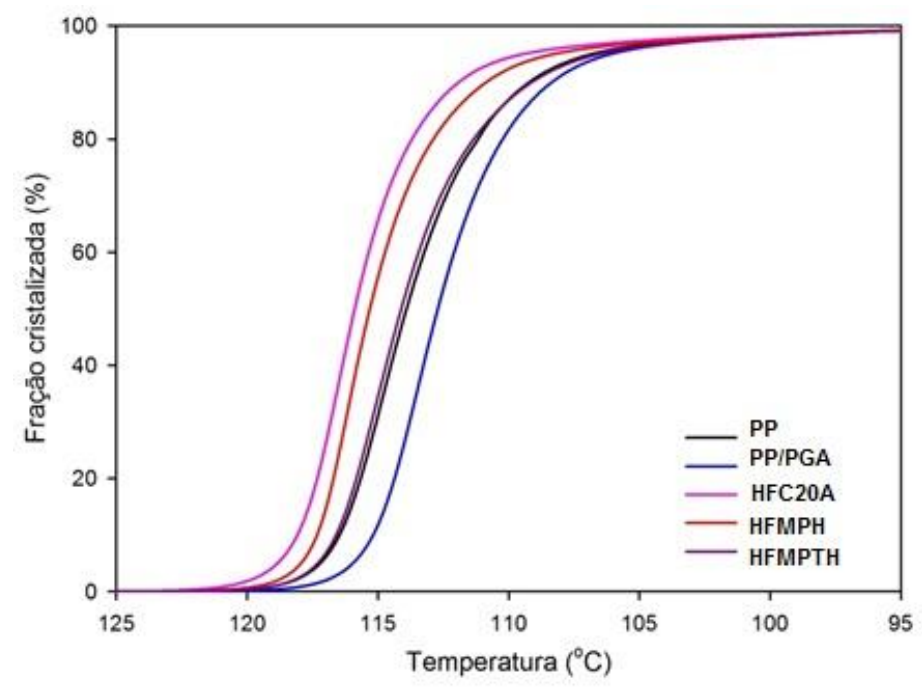

Figura 3. Grau relativo de cristalinidade (fração cristalizada) com a temperatura sob taxa de resfriamento de $10^{\circ} \mathrm{C} / \mathrm{min}$.

A Figura 4 mostra as curvas da taxa de cristalização em função da temperatura para o PP puro, PP/PGA e para os nanocompósitos (HFC20A, HFMPH e HFMPTH). Embora as duas argilas (MPH e MPTH), organofilizadas no laboratório de físicoquímica da Unidade Acadêmica de Mineração e Geologia da UFCG, possam ser usadas como nucleantes heterogêneos para o PP, a argila MPH é a mais indicada para tal finalidade, pois apresentaram um melhor efeito na nucleação do PP/PGA (Tabela 2). Com a adição de apenas 1\% em massa de MPH ao PP/PGA, a temperatura de cristalização do mesmo aumento de $112^{\circ} \mathrm{C}$ para $115^{\circ} \mathrm{C}$; próxima à apresentada pelo nanocompósito preparado com a argila organofílica comercial (C20A), proveniente da Southern Clay Products/EUA, que foi de $116^{\circ} \mathrm{C}$.

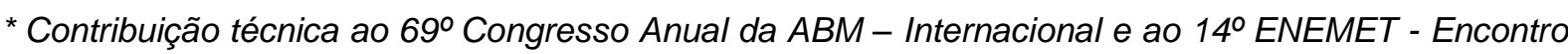
Nacional de Estudantes de Engenharia Metalúrgica, de Materiais e de Minas, 21 a 25 de julho de 2014, São Paulo, SP, Brasil.
} 

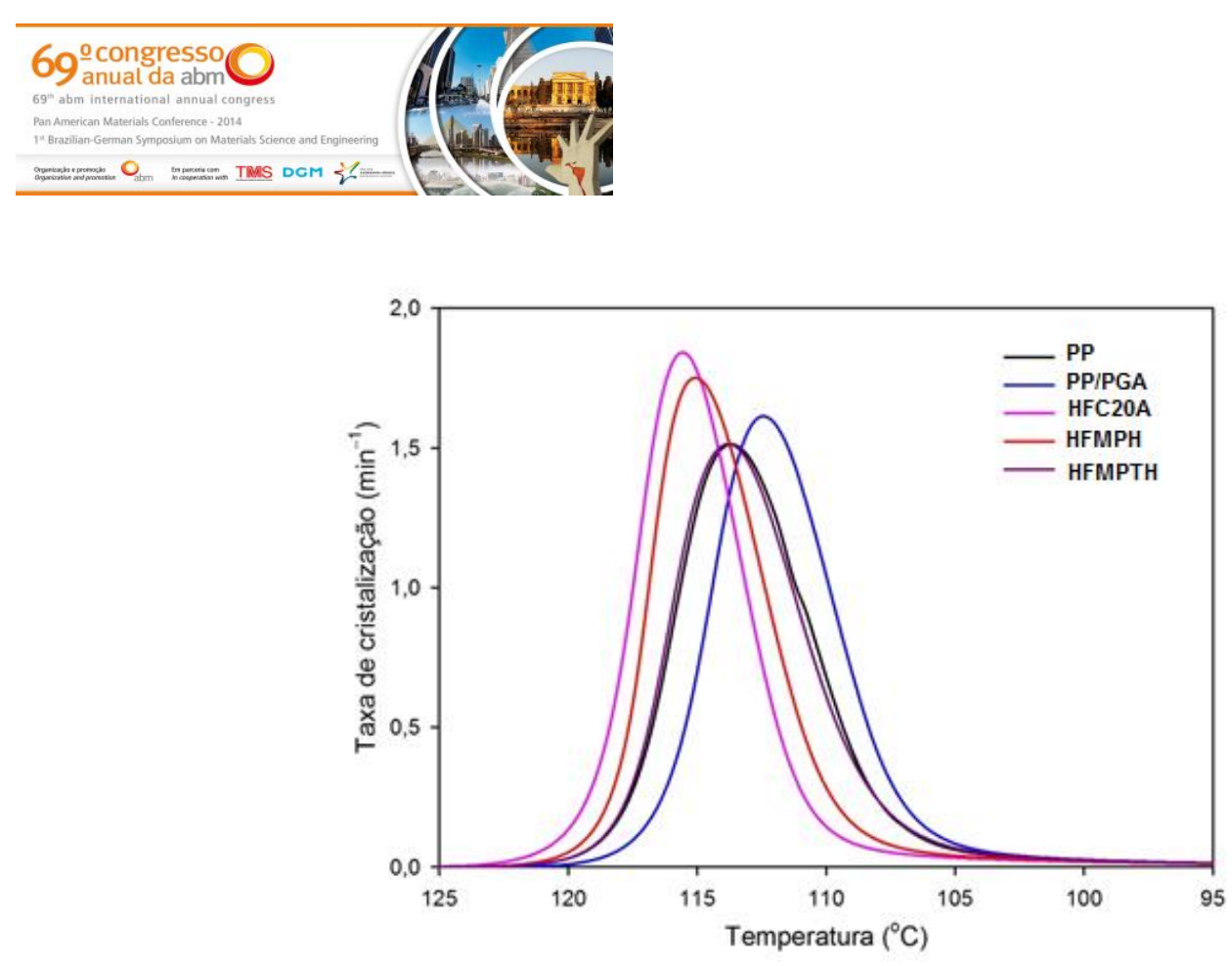

Figura 4. Taxa de cristalização com a temperatura sob taxa de resfriamento de $10^{\circ} \mathrm{C} / \mathrm{min}$.

Este é um resultado relevante do ponto de vista industrial, pois o PP é um polímero cristalizável, mas com velocidade de cristalização relativamente baixa. $\mathrm{O}$ aumento na velocidade de cristalização do PP garante uma solidificação mais rápida do polímero fundido quando resfriado. Isto leva a uma redução do tempo de ciclo durante a moldagem por injeção e, propriedades tais como resistência a tração, temperatura de distorção térmica e dureza são aumentadas pela ação do agente nuclenate. Por causa da diminuição no tamanho dos esferulitos, as propriedades óticas, como transparência e brilho do polímero nucleado são também melhoradas [22]. Além destas vantagens, como a argila proveniente de empresa local (Bentonit União Nordeste/Campina Grande/Paraíba), organofilizada com tecnologia local (MPH) apresentou comportamento semelhante ao da argila organofílica comercial (C20A), fornecida pela Southern Clay Products/ EUA, o uso destas (MPH) pode ser uma alternativa atraente para aplicações do PP moldado por injeção onde o custo é um fator primordial.

\section{CONCLUSÃO}

De acordo com os resultados obtidos fica evidenciado que o tipo de argila organofílica empregada na preparação dos híbridos afetou a morfologia e a cristalização do PP. Os dados de difratometria de raios $X$ evidenciam a formação de nanocompósitos com morfologia intercalada desordenada para o híbrido HFC20A e morfologia intercalada desordenada tendendo a esfoliada para os híbridos HFMPH e HFMPTH. As argilas organofílicas C20A, MPH e MPTH quando misturadas ao PP atuaram como agentes nucleantes heterogêneos para o PP, aumentando sua temperatura de cristalização. Os resultados obtidos mostraram que embora as duas argilas preparadas com tecnologia nacional (MPH e MPTH) tenham atuado como agente nucleante para o PP a MPH foi a mais eficiente, apresentando valores próximoas aos obtidos com a argila comercial.

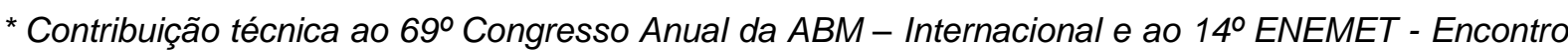
Nacional de Estudantes de Engenharia Metalúrgica, de Materiais e de Minas, 21 a 25 de julho de 2014, São Paulo, SP, Brasil.
} 


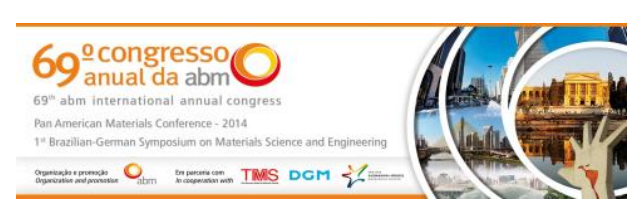

\section{Agradecimentos}

Os autores agradecem a Capes e ao CNPq pelo apoio financeiro a Bentonit União Nordeste S/A pela doação da bentonita in natura, à Felinto Indústria e Comércio pela doação do polipropileno, à Chemtura Indústria Química do Brasil pelo fornecimento do compatibilizante e ao prof. Dr. Marcus Vinícius Lia Fook por disponibilizar os equipamento do Certibio para caracterização das amostras.

\section{REFERÊNCIAS}

1 Twede D, Goddard R. Materiais para embalagens. São Paulo: Editora Blucher; 2010.

2 Modesti M, Lorenzetti A, Bon D, Besco S. Thermal 8ehavior of compatibilised polypropylene nanocomposite: Effect of processing conditions. Polymer Degradation and Stability. 2006; 91; 672-680.

3 Ray SS, Okamoto M. Polymer/layered silicate nanocomposites: a review from preparation to processing. Progress in Polymer Science. 2003; 28: 1539-1641.

4 Lei SG. Formulation and mechanical properties of polypropylene nanocomposites. M.Asc thesis, Concordia University, Department of Mechanical and Industrial Engineering; 2003.

5 Dong Yu, Bhattacharyya Debes. Effects of clay type, clay/compatibiliser content and matrix viscosity on the mechanical properties of polypropylene/organoclay nanocompósitos. Composites: Part A. 2008; 39: 1177-1191.

6 Liu X, He A, Du K, Han Charles C. Isothermal crystallization behavior of exfoliatedPP/IMMT nanocomposites via in situ polymerization. J Polym Sci Part B: Polym Phys. 2009; 47:2215-2225.

7 Maiti P, Nam PH, Okamoto M, Kotaka T, Hasegawa N, Usuki A. The effect of crystallization on the structure and morphology ofpolypropylene/clay nanocomposites. Polym Eng Sci. 2002; 42: 1864-1871.

8 Dai Xin, Zhang Zishou, Wang Chunguang, Ding Qian, Jiang Juan, Mai Kancheng. A novel montmorillonite with $b$-nucleating surface for enhancing b-crystallization of isotactic polypropylene Composites: Part A. 2013; 9:1-8.

9 Davis $\mathrm{CH}$, Mathias LJ, Gilman JW, Schiraldi DA, Shields JR, Trulove P, Sutto TE, Delong HC. Effects of melt-processing conditions on the quality of Poly(ethylene terephthalate) montmorillonite clay nanocomposites. Journal of Polymer Science: Part B: Polymer Physics. 2002; 40: 2661-2666.

10 García-López D, Picazo O, Merino JC, Pastor JM. Polypropylene-clay nanocomposites: effect of compatibilizing agents on clay dispersion. European Polymer Journal. 2003; 39(5): 945-950.

11 Tidjani A, Wald O, Pohl M, Hentschel MP, Schartel B. Polypropylene-graft-maleic anhydride-nanocomposites: I- Characterization and thermal stability of nanocomposites produced under nitrogen and in ai. Polymer Degradation and Stability. 2003; 82: 133140.

12 Coelho ACV, Souza Santos P. Argilas especiais: argilas quimicamente modificadas Uma revisão. Química Nova. 2007; 30: 1282-1294.

13 Ficha Técnica do Produto Southern Clay Products. Disponível em: $<$ http://www.scprod.com/product_bulletins.asp>.

14 Ficha Técnica do produto Quattor. Disponível em: <http://www.quattor.com.br/quattor web/pt/index.aspx>.

15 Ficha Técnica do Produto Chemtura Indústria Química do Brasil. Disponível em: $<$ http://www.specialchem4polymers.com/tds/polybond3150/chemtura/9374/index.aspx?! r=google\&gclid=COnr5dSj7>.

\footnotetext{
* Contribuição técnica ao $69^{\circ}$ Congresso Anual da ABM - Internacional e ao 14ํㅡㄹ ENEMET - Encontro Nacional de Estudantes de Engenharia Metalúrgica, de Materiais e de Minas, 21 a 25 de julho de 2014, São Paulo, SP, Brasil.
} 


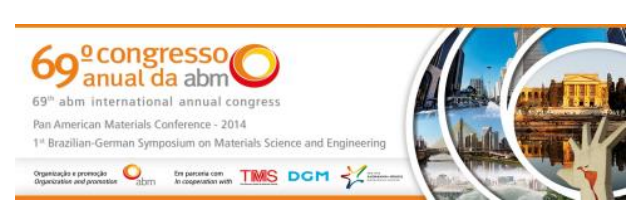

16 Morelli FC, Ruvolo Filho A. Nanocompósitos de Polipropileno e Argila Organofílica: Difração de Raios X, Espectroscopia de Absorção na Região do Infravermelho e Permeação ao Vapor D'água. Polímeros: Ciência e Tecnologia. 2011; 20 (2): 121-125.

17 Lertwilmolnun W, Vergnes B., Influence of compatibilizer and processing conditions on the dispersion of nanoclay in a polypropylene matrix. Polymer. 2005; 46: 3462.

18 de Paiva LB, Morales AR, Guimarães TR. Propriedades mecânicas de nanocompósitos de polipropileno e montmorilonita organofílica. Polímeros Ciência e Tecnologia. 2006;16(2):136.

19 Fermino DM. Estudo das propriedades mecânicas, reológicas e térmicas de nanocompósito de HMSPP (polipropileno com alta resistência do fundido) com uma bentonita Brasileira: Universidade de São Paulo. São Paulo; 2011.

20 Leite IF, Malta OL, Raposo CM, Canedo LE, Carvalho LHd, Silva SMdL. Effect of different types of clays and organic modifiers on the morphology and thermal properties of PET nanocomposites. Polímeros. 2011;21(3):195-203.

21 Calcagno CIW, Mariani CM, Teixeira SR, Mauler RS. The effect of organic modifier of the clay on morphology and crystallization properties of PET nanocomposites. Polymer. 2007; 48: 966-974.

22 Wang Y, Gao J, Ma Y, Agarwal US. Study on mechanical properties, thermal stability and crystallization behavior of PET/MMT nanocomposites Composites: Part B. 2006; 37:399-407.

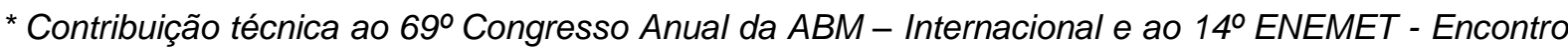
Nacional de Estudantes de Engenharia Metalúrgica, de Materiais e de Minas, 21 a 25 de julho de 2014, São Paulo, SP, Brasil. 